\title{
Propagation d'ondes en milieu centrifugé : caractérisation, dispersion, atténuation
}

\section{J.-F. SEMBLAT}

Laboratoire Central des Ponts et Chaussées.

58, bd Lefebvre

75732 Paris Cedex, 15

semblat@Icpc.fr

\section{M.P. LUONG}

Laboratoire de Mécanique des Solides,

Ecole polytechnique,

91128 Palaiseau Cedex luong@athena.polytechnique.ff

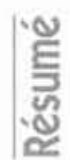

Les phénomènes de propagation d'ondes dans les sols peuvent être simulés expérimentalement sur modèles récuits centrifugés. Deux types de sollicitation sont utilisés : simulation de sêismes, chute de masse sphérique en surface. La propagation est analysée en termes de lois de dispersion. Il est tenu compte des réflexions d'ondes sur les parois (élimination par filtrage homomorphique). Pour les essais de chute de masse, l'analyse du champ d'ondes sphériques en viscoélasticité linéaire conduit à une description analytique complète de la propagation.

Wave propagation in centrifuged medium : characterization, dispersion, damping

Wave propagation phenomena in solls can be experimentally simulated on centrifuge scale models. There are two types of excitation devices : earthquake simulations, drop-ball arrangement. Propagation is investigated through dispersion laws. Wave reflections on model boundaries are taken into account and removed by homomorphic filtering. For drop-ball tests, spherical wave field analysis assuming linear viscoelasticity leads to a complete analytical description of wave propagation. 


\section{Introduction}

Le comportement dynamique d'un sol peut rarement être appréhendé directement. Les approches sont nombreuses et variées : essais cycliques, méthodes sismiques, essais ultrasonores... Mais les fréquences et les déformations mises en jeu ne sont pas du même ordre. Une classification de ces différentes méthodes est proposée dans $[12,14]$ qui tient compte en outre du rapport $\lambda / 1_{\text {reff }}$ longueur d'onde sur taille du domaine d'étude (ou de l'échantillon). La sollicitation dynamique ou vibratoire d'un sol induit toujours des phénomènes de propagation. Leur importance dépend de ce rapport $\lambda 1_{\text {rér. }}$ Dans cet article ces phénomènes sont largement prépondérants et mettent en jeu des questions particulières : dispersion, atténuation... Ce texte présente quelques résultats d'expériences de propagation d'ondes dans un massif de sol centrifugé.

Les expérimentations dynamiques en massif centrifugé réalisées ces dernières années [2, 3, 4] sont particulièrement pertinentes pour interpréter les problèmes sismiques. Les principales préoccupations des expérimentateurs sont les suivantes

- améliorer la représentativité de la source d'excitation tout en conservant une certaine simplicité de mise en œuvre (cet aspect est primordial pour les expérimentations en centrifugeuse)

- s'affranchir au maximum des problèmes de réflexion d'ondes qui, dans un massif de dimensions finies, peuvent avoir des conséquences graves sur l'analyse des résultats (lois de dispersion, atténuation).

\section{2}

\section{Expériences réalisées}

\section{Dispositif expérimental}

Les expérimentations en centrifugeuse réalisées dans cette étude utilisent plusieurs types de sollicitations transitoires : simulations de séismes par explosifs, création d'ondes par chute de masse sphérique en surface (voir Fig. 1). Le massif de sol centrifugé est constitué de sable de Fontainebleau sec, Ses dimensions sont les suivantes: longuew $L=1,30 \mathrm{~m}$, largeur $\mathrm{I}=0,80 \mathrm{~m}$ et hauteur $\mathrm{h}=0,38 \mathrm{~m}$.

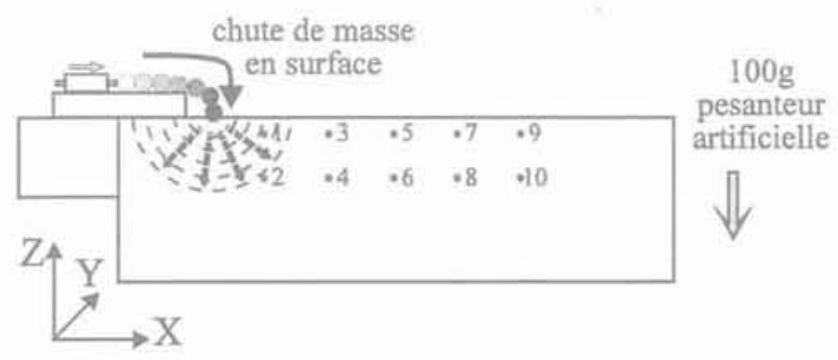

FIG.1 Dispositif expérimental pour les essais de chute de masse.

Drop-ball arangement for centrifuge experiments.
Les méthodes de simulation de séismes par explosifs produisent des sollicitations d'assez longue durée. Le contenu fréquentiel est assez riche (Fig. 2), et l'énergie mise en jeu peut être contrôlée de façon précise. Toutefois, des phénomènes parasites d'interaction entre le dispositif d'excitation et le caisson contenant le massif centrifugé peuvent intervenir. Cela rend délicate l'étude des phénomènes de réflexion, de dispersion ou d'atténuation.

Le dispositif expérimental présenté à la figure 1 crée une sollicitation de courte durée (Fig. 3 et aussi [1, 12]), et il n'y a pas transmission d'une partie des ondes au conteneur lui-même. Des accéléromètres tridimensionnels sont placés dans le massif centrifugé ( 5 près de la surface et 5 à mi-profondeur) afin de mesurer les composantes des vecteurs accélérations suivant les trois directions de l'espace (Fig. 1).

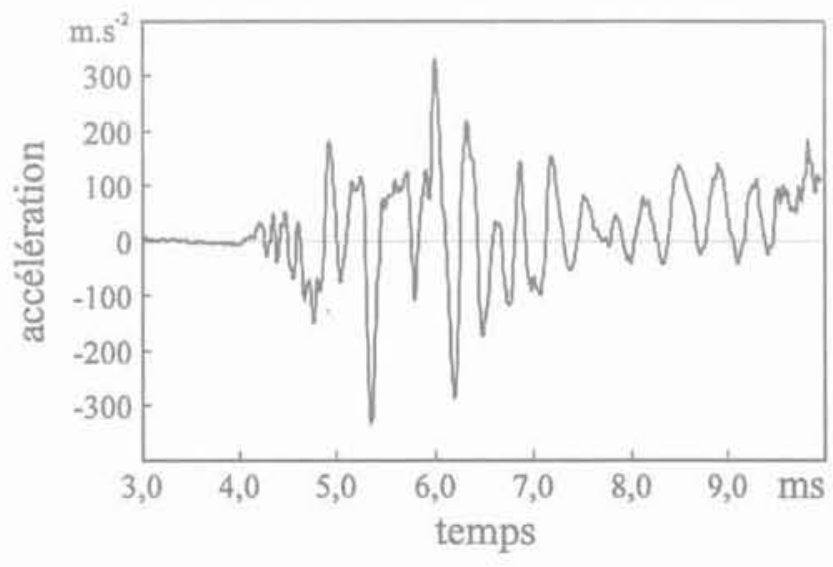

FiG.2 Signal d'accélération pour un essai de simulation de séisme.

Acceleration signal for earthquake simulator experiment.

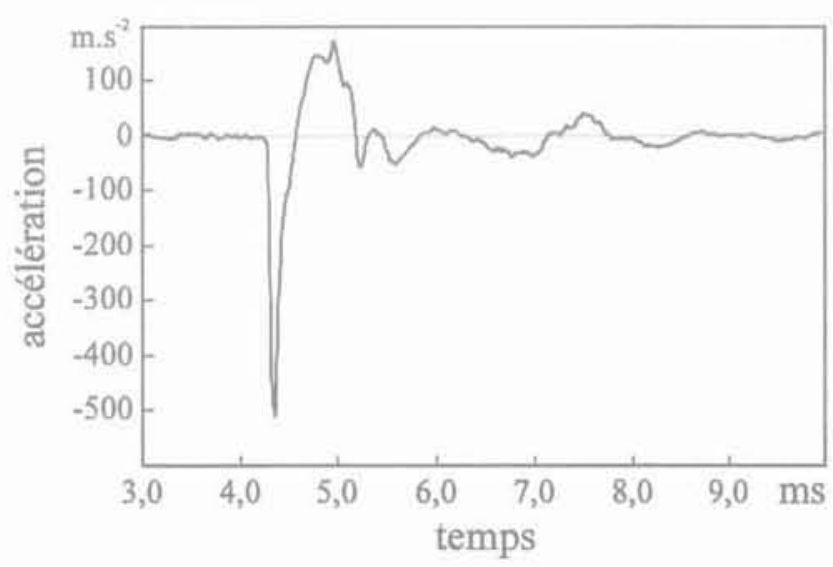

F日. 3 Signal d'accélération pour un essai de chute de masse. Acceleration signal for drop-ball experiments.

La sollicitation créée à l'aide du dispositif de la figure 1 permet une étude précise des phénomènes de réflexion (les différentes ondes peuvent ètre parfaitement distinguées) et autorise une analyse fine de l'atténuation des ondes. 
Les distances des différents accéléromètres par rapport au point d'impact de la masse sphérique sont données dans le tableau I. Ces distances entre les accéléromètres et la source d'ondes sphériques sont utilisées dans la suite pour la quafication des vitesses et des amortissements.

TABEEAU: Distance des différents capteurs par rapport à la source d'ondes sphériques. Distance from the sensors to the spherical wave source.

\begin{tabular}{l|c|c|c|c|c}
\hline \multicolumn{7}{c}{ Capteurs de surface } \\
\hline Numéro & 1 & 3 & 5 & 7 & 9 \\
\hline Distance & $0,11 \mathrm{~m}$ & $0,24 \mathrm{~m}$ & $0,37 \mathrm{~m}$ & $0,50 \mathrm{~m}$ & $0,63 \mathrm{~m}$ \\
\hline \multicolumn{6}{c}{ Capteurs de profondeur } \\
\hline Numéro & 2 & 4 & 6 & 8 & 10 \\
\hline Distance & $0,19 \mathrm{~m}$ & $0,28 \mathrm{~m}$ & $0,40 \mathrm{~m}$ & $0,52 \mathrm{~m}$ & $0,65 \mathrm{~m}$ \\
\hline
\end{tabular}

\section{2}

\section{Caractérisation des ondes}

Les mesures d'accélération tridimensionnelles réalisées donnent les vecteurs accélération dans l'espace $\mathrm{XYZ}$. Les composantes suivant l'axe Y (Fig. 1), c'est-àdire perpendiculairement au plan de propagation, sont quasiment nulles. La figure 4 donne les valeurs d'accélération dans le plan XZ pour un essai de chute de masse : capteurs de surface en haut (numéros impairs) et capteurs de profondeur en bas (numéros pairs). Ces courbes permettent de déterminer la direction et l'amplitude des vecteurs accélération à différents instants.
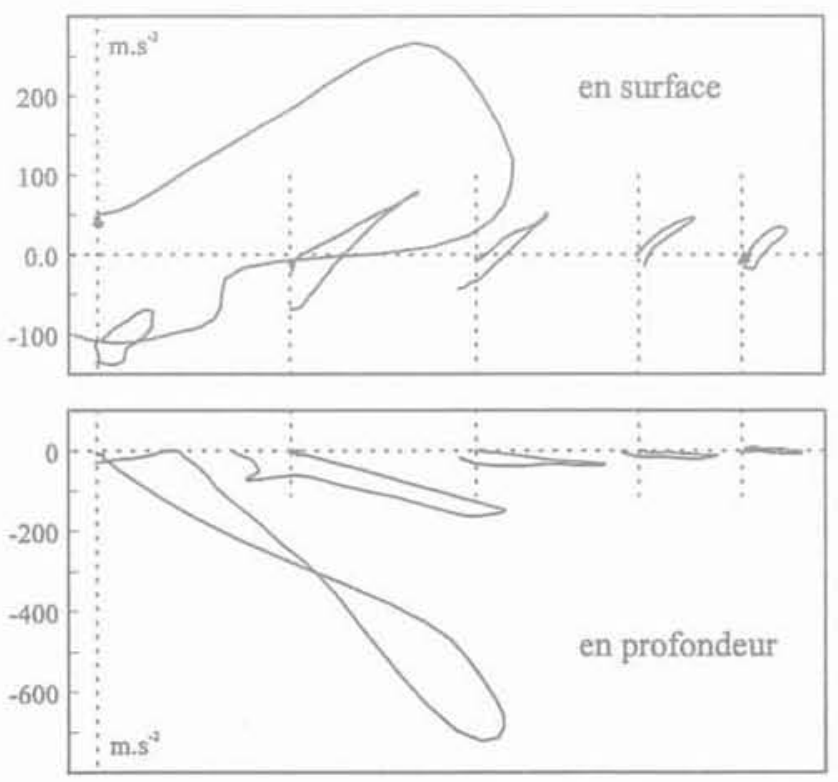

FiG.4 Accélérations dans le plan XZ d'après mesures expérimentales.

Acceleration in $\mathrm{XZ}$ plane from experimental results.
Les vecteurs accélérations correspondant à l'amplitude maximale sont déterminés d'après les résultats de la figure 4. Ils sont représentés sur le schéma de la figure 5 . Pour les capteurs situés en profondeur, la direction d'incidence est identique à la direction d'accếlération. Pour tous ces capteurs, le mouvement se fait donc suivant la direction de propagation. Il est alors possible de conclure que, à mi-profondeur, les ondes mesurées sont essentiellement des ondes longitudinales (Fig. 5). Les valeurs des angles d'incidence et des directions des vecteurs accélération sont données dans [12].

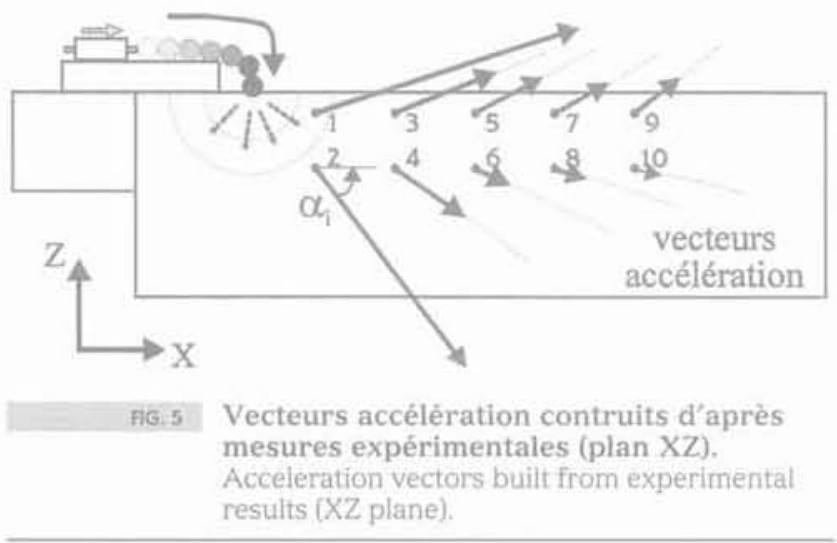

\section{Quelques résultats sur la propagation}

\section{1}

\section{Vitesses de propagation}

Une première évaluation de la vitesse de propagation est réalisée soít directement en mesurant les délais entre pics sur les signaux temporels issus des différents accéléromètres soit à l'aide de diverses techniques de traitement du signal [12] (fonctions d'intercorrélation, phase temporelle), Une évaluation plus fine des vitesses de phase et de groupe sera proposée plus loin grâce à une analyse temps-fréquence des signaux d'accélération.

Les valeurs obtenues pour les essais de simulation de séismes donnent une vitesse de propagation moyenne d'environ $500 \mathrm{~m} \cdot \mathrm{s}^{-1}$ [12]. Pour les essais de chute de masse, les valeurs de vitesse correspondant aux différents capteurs sont données dans le tableau Il (essai n ${ }^{\circ} 13$ ).

\begin{tabular}{|c|c|c|c|c|}
\hline \multirow[t]{2}{*}{ TABLEAU If } & \multicolumn{4}{|c|}{$\begin{array}{l}\text { Vitesses de propagation, essai de chute de } \\
\text { masse } n^{*} 13 \text {. } \\
\text { Wave velocities for drop-ball test } n^{*} 13 \text {. }\end{array}$} \\
\hline & \multicolumn{4}{|c|}{ Vitesses de propagation } \\
\hline \multirow{2}{*}{ Surface } & $1 / 3$ & $3 / 5$ & $5 / 7$ & $7 / 9$ \\
\hline & $433 \mathrm{~m} / \mathrm{s}$ & $400 \mathrm{~m} / \mathrm{s}$ & $426 \mathrm{~m} / \mathrm{s}$ & $448 \mathrm{~m} / \mathrm{s}$ \\
\hline \multirow{2}{*}{ Profondeur } & $2 / 4$ & $4 / 6$ & $6 / 8$ & $8 / 10$ \\
\hline & $409 \mathrm{~m} / \mathrm{s}$ & $421 \mathrm{~m} / \mathrm{s}$ & $429 \mathrm{~m} / \mathrm{s}$ & $419 \mathrm{~m} / \mathrm{s}$ \\
\hline
\end{tabular}


Le tableau II donne les valeurs de vitesses de propagation calculées d'après les mesures d'accélération en surface et en profondeur pour l'essai de chute de masse $n^{\circ} 13$. La valeur moyenne de la vitesse de propagation pour cet essai est $v_{\text {mon }}=423 \mathrm{~m} \cdot \mathrm{s}^{-1}$. D'après le tableau II, II n'y a pas de différence notable entre vitesses en surface et vitesses en profondeur. Il est à noter que les valeurs de vitesse calculées pour les essais de chute de masse sont plus faibles que les valeurs issues des essais de simulation de séismes.

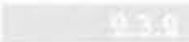

\section{Simulations numériques simples}

Quelques résultats numẻriques simples (élasticité linéaire) sont proposés à la figure 6. Iis permettent d'illustrer les principales caractéristiques de la propagation des ondes dans le massif centrifugé: type d'ondes, réflexions au fond du massif... Ces calculs ont été réalisés avec le progiciel de calcul par éléments finis CESAR-LCPC à partir d'une modélisation 2D-axisymétrique. Les résultats proposés à la figure 6 correspondent à quatre valeurs de temps différentes :

- temps $T_{1}$ : début de la propagation, les différents types d'ondes ne peuvent encore ètre distingués ; - temps $T_{2}$ : séparation claire des ondes longitudinales (les plus rapides), des ondes de cisaillement et des ondes de surface;

- temps $T_{3}$ : début de la réflexion des ondes longitudinales au fond du massif :

- temps $T_{4}$ : les ondes longitudinales et de cisaillement continuent à se propager alors que l'onde P réfléchie se propage vers la surface libre.

D'après les résultats expérimentaux des figures 4 et 5 , il apparait clairement que seules les ondes longitudinales sont détectées par les accéléromètres situés à miprofondeur. En revanche, comme l'indiquent les résultats expérimentaux et numériques (Fig. 4, 5, 6), plusieurs types d'ondes sont présents en surface.

Des modélisations numériques plus complexes sont en cours afin d'analyser de façon quantitative les résul- tats expérimentaux [15, 16, 17]. Toutefois, sont proposés dans la suite des modèles analytiques pour étudier et quantifier avec précision les phénomènes d'atténuation et de dispersion ( $\$ 5$ et 6).

\section{4}

\section{Conditions de similitude en dynamique}

L'expérimentation sur modèles réduits nécessite le respect de conditions de similitude entre le prototype en grandeur réelle et le modèle réduit centrifugé. Les essais en centrifugeuse rendent compte de façon réaliste du comportement des matériaux et des ouvrages car les contraintes sont identiques dans le modèle réduit centrifugé malgré la réduction des dimensions. Ceci est rendu possible par l'utilisation des forces de pesanteur artificielles supérieures à celles subies par le prototype (ce sont les équations de la mécanique qui imposent cette condition).

\begin{tabular}{c|c|c}
\hline \multicolumn{2}{|c|}{$\begin{array}{c}\text { TABLEAUII } \\
\text { Scaling relations for dynamic experiments }\end{array}$} \\
$\begin{array}{c}\text { Grandeur } \\
\text { mécanique }\end{array}$ & $\begin{array}{c}\text { Rapport de } \\
\text { similitude }\end{array}$ & $\begin{array}{c}\text { Valeur } \\
\text { numérique }\end{array}$ \\
\hline Longueur & $\mathrm{r}^{*}$ & $1 / 100$ \\
\hline Temps & $\mathrm{t}^{*}$ & $1 / 100$ \\
\hline Vitesse & $\mathrm{v}^{*}$ & 1 \\
\hline Fréquence & $\mathrm{f}^{*}$ & 100 \\
\hline Accélération & $\mathrm{a}^{*}$ & 100 \\
\hline Viscosité & $\eta^{*}$ & $1 / 100$ \\
\hline Amortissement & $\alpha^{*}$ & 100 \\
\hline
\end{tabular}

Dans les essais réalisés, le massif de sable est centrifugé à $100 \mathrm{~g}$ (g : accélération de la pesanteur) et les rapports de similitude en longueur, temps, vitesse sont
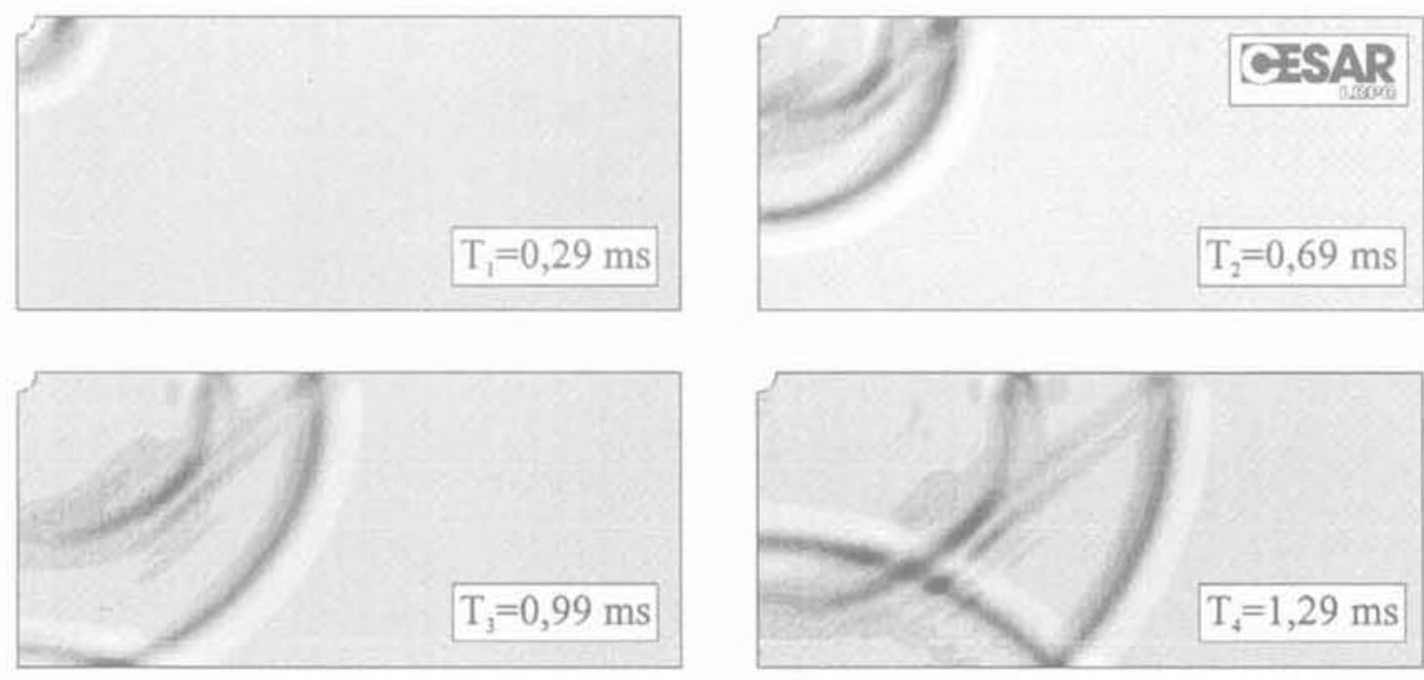

FG. 6 Simulations numériques de la propagation d'ondes sphériques dans le plan $\mathrm{XZ}$ (CESAR-LCPC).

Numerical modelling of spherical wave propagation in XZ plane (CESAR-LCPC). 
donnés dans le tableau III. En dynamique, il est nécessaire de respecter la similitude du point de vue de l'excitation et des phénomènes de propagation. Il faut donc respecter les rapports de similitude en amplitude d'accélération, en fréquence et pour les phénomènes d'atténuation en viscosité ou en amortissement.

Il est indispensable d'examiner un autre aspect important des essais sur modèles réduits. Ces essais sont, par définition, réalisés sur des milieux de dimensions finies. La propagation d'ondes dans le massif est perturbée par les réflexions d'ondes sur les frontières du massif. Cela influence l'analyse de la dispersion, de l'atténuation..

\section{3}

\section{Réflexions d'ondes}

\section{$3: 1$}

\section{Détection}

La réalisation d'expériences de propagation d'ondes en milieu fini se heurte à une difficulté majeure : la propagation des ondes est rapidement entravée du fait des dimensions du massif. La figure 7 donne les signaux d'accélération (module du vecteur) mesurés par les capteurs de profondeur pour un essai de chute de masse. Les réflexions d'ondes en fond de massif peuvent être repérées directement sur ces signaux. Dans certains cas, il est nécessaire d'utiliser des techniques particulières pour détecter avec précision les ondes réfléchies : fonction d'autocorrélation, cepstre réel, phase temporelle [12].

Les résultats expérimentaux obtenus en centrifugeuse [12] montrent que les réflexions d'ondes en fond de massif sont très importantes (Fig. 7). L'utilisation du dispositif expérimental de la figure 1 permet une détection aisée des ondes réfléchies. Comme le train d'onde incident est de durée assez courte, il est possible de déterminer, pour chaque capteur, les délais entre onde directe et onde réfléchie [12].

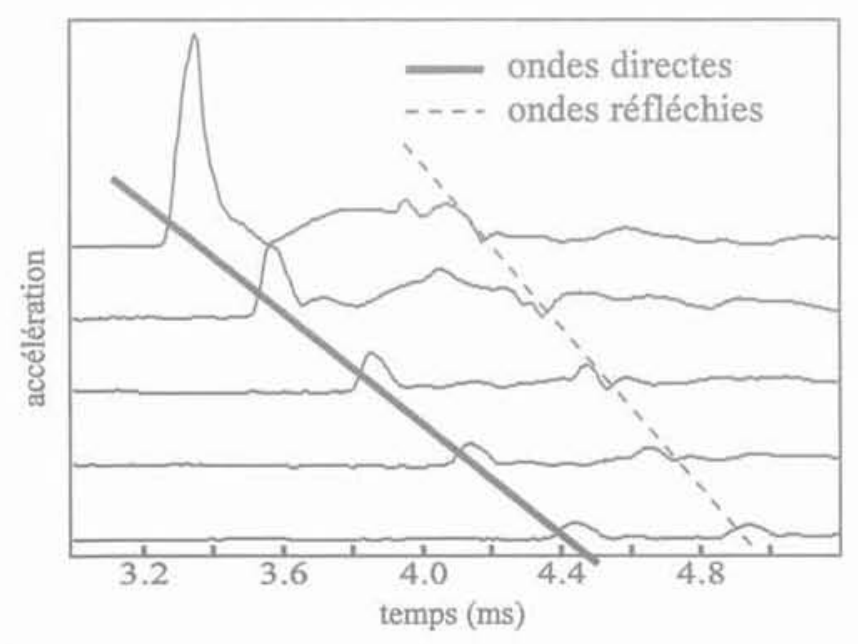

คG.7 Signaux d'accélération (module du vecteur) à mi-profondeur.

Accelerations signals for mid-depth sensors.

\section{Élimination}

Certains auteurs proposent de limiter les réflexions d'ondes sur les parois à l'aide d'un produit isolant [4]. Dans cette étude, une autre approche est proposée qui consiste à éliminer les ondes réfléchies par filtrage homomorphique (après calcul du cepstre complexe).

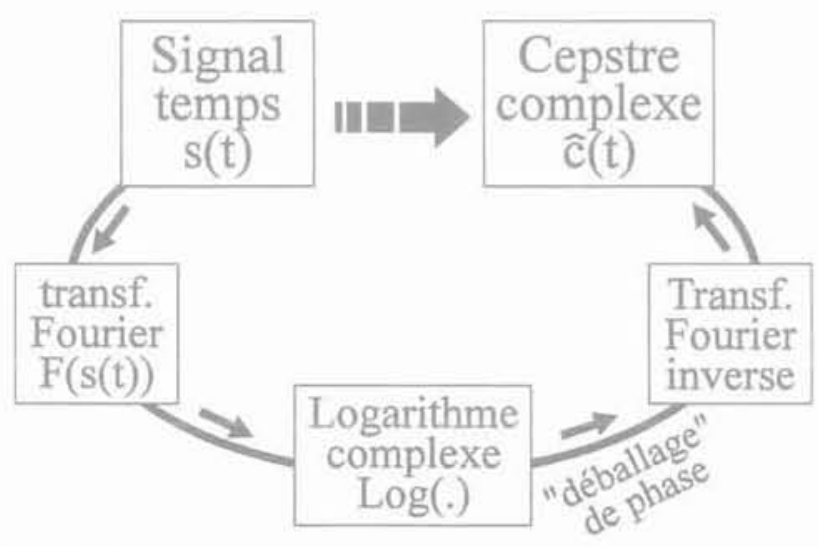

nG. \& Principe de calcul du cepstre complexe. Complex cepstrum : basic principles.

Cette méthode est difficile à mettre en œuvre mais s'avère très efficace y compris pour des sollicitations de durée importante. La méthode utilisée consiste à calculer le cepstre complexe du signal d'accélération [12, 18, 19]. Le principe de ce calcul du cepstre complexe est présenté à la figure 8 .

L'étape la plus délicate dans le calcul du cepstre complexe est le $\alpha$ déballage $*$ de la phase, c'est-à-dire l'opération qui consiste à rendre la phase continue. Cette étape est menée à bien grâce à un algorithme proposé par Tribolet [18].

L'algorithme proposé par Tribolet consiste à calculer la dérivée de la phase à l'aide de l'expression suivante :

$$
\frac{d(\operatorname{Arg}(X(f))}{d f}=\frac{X_{R}(f) \cdot X_{1}^{\prime}(f)-X_{1}(f) \cdot X_{R}^{\prime}(f)}{|X(f)|^{2}}
$$

Il suffit alors d'intégrer le résultat pour obtenir la phase continue qui permet de calculer le cepstre complexe du signal. Cette fonction présente des pics caractéristiques de l'arrivée d'échos ou d'ondes réfléchies. L'utilisation d'une fenêtre de pondération permet d'éliminer ces échos dans le domaine * cepstral ») (opération appelée « liftrage n). Cette technique est très intéressante car l'élimination est aussi possible pour une sollicitation de longue durée (onde directe et réfléchie(s) mêlées).

Pour les signaux d'accélération mesurés expérimentalement, ce type de traitement a été réalisé. La figure 9 montre que, pour un essai de chute de masse, le filtrage homomorphique permet d'éliminer l'onde réfléchie en fond de massif. 


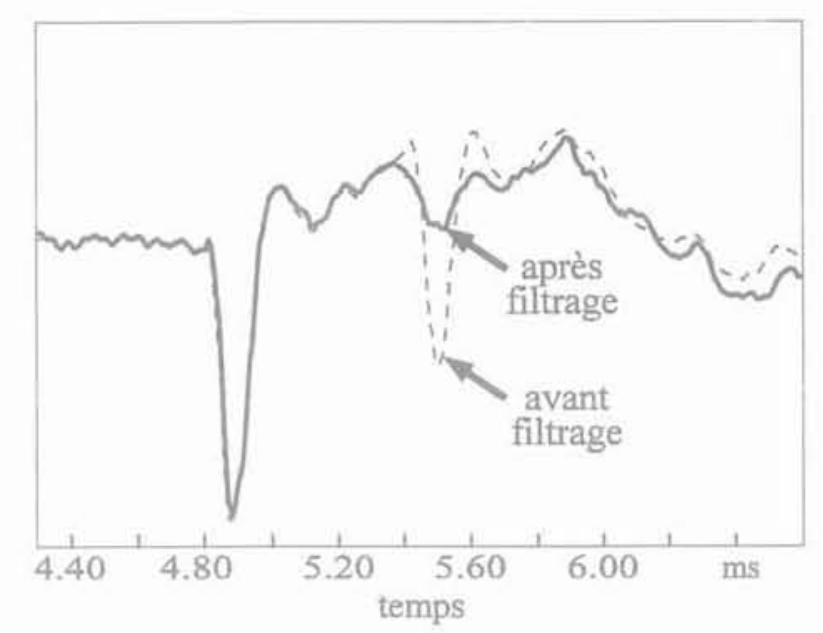

FG. 9 Élimination d'échos par filtrage homomorphique.

Echoes removal by homomorphic filtering.

\title{
Phénomènes de dispersion
}

\section{1}

\section{Analyse temps-fréquence}

\author{
Itath \\ Intérêt
}

L'étude de la propagation proprement dite passe par la connaissance des lois de dispersion et l'analyse des phénomènes d'atténuation. La décomposition des signaux d'accélération dans le domaine temps-fréquence donne les caractéristiques dispersives des phénomènes de propagation observés $[6,8,12]$. Cette analyse peut ètre réalisée à l'aide de différentes méthodes: filtrage passe-bande, transformation en ondelettes... Dans les différentes bandes de fréquence explorées, les lois de dispersion (vitesses de phase et de groupe) dans le milieu considéré peuvent ainsi être déterminées.

\section{That}

\section{Distribution de Wigner-Ville}

La distribution de Wigner-Ville donne une approche théorique globale de l'analyse des signaux dans le domaine temps-fréquence [6]. Le principal avantage de cette méthode est qu'elle laisse une liberté totale de choix des fonctions de pondération en temps et en fréquence. La distribution de Wigner-Ville offre la possibilité, dans le cas de signaux fortement dispersifs, d'accéder à la mesure de la vitesse de groupe.

\section{5}

\section{Transformation en ondelettes}

La distribution de Wigner-Ville comprend donc dans sa définition de nombreux autres types de trans-

formations : transformée de Fourrier à fenêtre glissante, transformée en ondelettes... La transformation en ondelettes consiste à construire une famille de fonctions élémentaires $\psi_{\text {ab }}(\mathrm{t})$ définies à partir d'une ondelette-mère ou ondelette analysante $\psi(t)[6,8,12]$. Cette famille de fonctions s'écrit sous la forme suivante :

$$
\psi_{a b}(t)=\frac{1}{\sqrt{a}} \cdot \psi\left(\frac{t-b}{a}\right), b \in \Re, a>0
$$

Les coefficients d'ondelette du signal s, soient $C_{s}(a, b)$, permettent de caractériser s dans le domaine temps-fréquence et s'écrivent sous la forme suivante

$$
C_{s}(a, b)=\left\langle s, \psi_{a b}\right\rangle=\int_{-\infty}^{+\infty} s(t) \cdot \bar{\psi}_{a b}(t) \cdot d t
$$

En faisant varier les paramètres a et b, l'ondelette analysante $\psi(t)$ est simultanément translatée (paramètre b) et dilatée (ou contractée, paramètre a). Le signal peut alors être analysé dans le domaine temps-fréquence par décomposition sur la famille d'ondelettes ainsi créée.

La figure 10 donne la forme de l'ondelette analysante utilisée dans le cadre de cette étude. Une famille d'une centaine d'ondelettes est alors contruite qui permet de déterminer les coefficients $C_{s}(a, b)$ et d'étudier ainsi les phénomènes de dispersion entre 500 et $9500 \mathrm{~Hz}$ environ (voir également [12]).

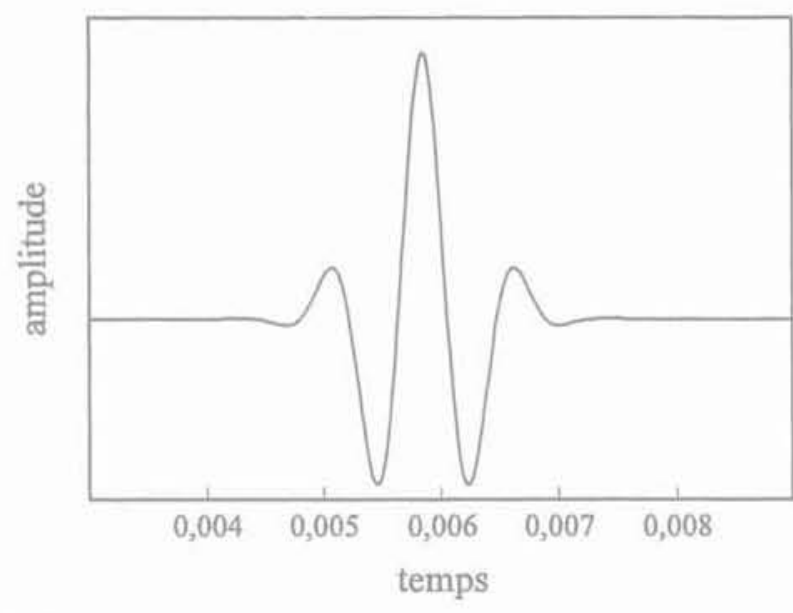

FIG. 70 Ondelette analysante utilisée pour l'analyse temps-fréquence des signaux d'accélération.

Analysing wavelet used for time-frequency analysis of acceleration signals.

\section{Étude de la dispersion}

La famille d'ondelettes décrite au paragraphe précédent (Fig. 10) permet d'analyser la propagation dans différentes bandes de fréquences. Il est alors possible de déterminer la vitesse de phase en confrontant, pour les différents capteurs, les signaux analysés à différentes fréquences (voir Fig. 11). 


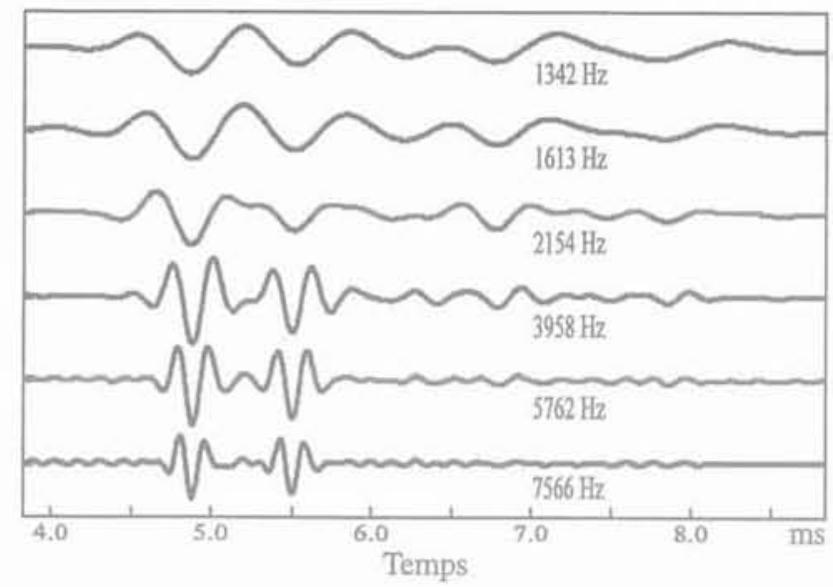

FlG. 11 Signaux d'accélération filtrés dans différentes bandes de fréquence. Acceleration signals filtered in different frequency bands.

La détermination de la vitesse de groupe se fait en calculant les courbes enveloppes des signaux filtrés (Fig, 11). La courbe enveloppe d'un signal est déterminée grâce à la transformée de Hilbert temporelle de ce signal $[5,12]$.

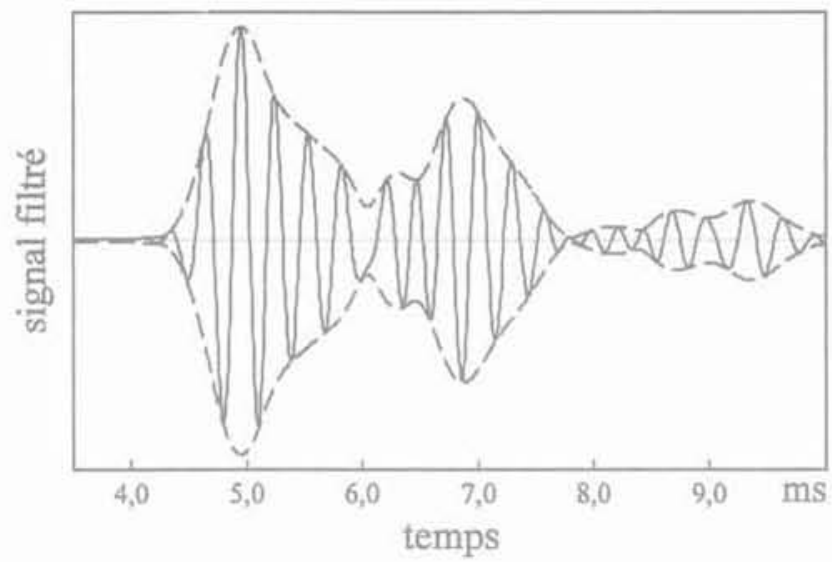

Fia. 12 Signal (accélération) filtré à $3175 \mathrm{~Hz}$ et courbe enveloppe associée.

Acceleration signal filtered at $3,175 \mathrm{~Hz}$ and its envelope curve.

Dans le domaine temporel, la transformée de Hilbert $h(t)$ d'un signal s(t) permet de séparer les informations de phase et d'amplitude afin de les étudier simultanément. La fonction complexe $\hat{S}(\mathrm{t})$ définie par $S(t)=s(t)+i . h(t)$ représente en effet l'enveloppe complexe du signal (ou " signal analytique »). Les informations d'amplitude sont données par le module de cette fonction $|S(t)|$ qui représente la courbe enveloppe.

La figure 12 donne un exemple de signal analysé (ou filtré) dans une bande de fréquence et de la courbe enveloppe correspondante. A partir des signaux des figures 11 et 12, les vitesses de phase et de groupe peuvent être déterminées. Plusieurs valeurs numériques sont données dans [12].

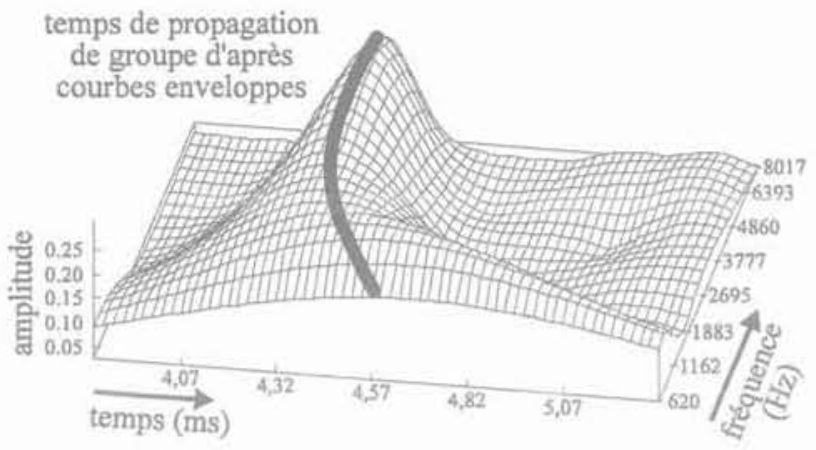

FG.13 Temps de propagation de groupe pour un essai de chute de masse.

Group delays for drop-ball experiment.

Un résultat pius qualificatif est proposé à la figure 13. Une série de courbes enveloppes correspondant à différentes fréquences est donnée pour un essai de chute de masse. Ces courbes indiquent que le temps de propagation de groupe augmente fortement aux basses fréquences et varié lentement aux fréquences moyennes et hautes.

Deux types de phénomènes dispersifs sont à distinguer (voir figure 13) :

- la dispersion géométrique qui est prépondérante pour les faibles fréquences (dimensions du massif petites devant la longueur d'onde : effet de guide d'ondes) ;

- la dispersion matérielle qui caractérise le milieu luimême pour les fréquences moyennes et hautes pour lesquelles il n'y a pas d'effet de guide d'ondes.

L'analyse des phénomènes de propagation (ici la dispersion) est donc largement influencée par la géométrie du modèle (milieu fini). La dispersion sera également analysée dans le paragraphe suivant au travers de quelques simulations simples.

\section{5}

\section{Ondes sphériques en milieu viscoélastique}

\section{1}

\section{Atténuation géométrique et matérielle}

\section{Silat}

\section{Atténuation géométrique}

Pour les essais de chute de masse, le champ d'ondes créé étant sphérique, la part de l'amortissement géométrique dans les phénomènes d'atténuation est grande, puisque l'amplitude est généralement considérée comme inversement proportionnelle à la distance $r$ de la source d'excitation. L'amplitude de l'onde d'accélération au point $M$ peut s'écrire : $A=A_{0} / r$. La comparaison des amplitudes d'accélération aux points $M$ et $M_{j}$ conduit à une expression très simple :

$$
A_{j}=A_{i} \cdot \frac{r_{1}}{r_{j}}
$$




\section{5 in: 9}

\section{Atténuation matérielle}

Les expériences en centrifugeuse réalisées par Luong dans les années 80 suggèrent une atténuation matérielle de la forme $a(x)=a_{0} \exp (-\alpha \cdot x)$, où le coefficient d'amortissement $\alpha$ est supposé indépendant de la fréquence et égal à $1,1 \mathrm{~m}^{-1}$. Pour les essais de chute de masse, en combinant atténuations géométrique et matérielle, la comparaison des amplitudes d'accélération en deux points différents s'écrit sous la forme suivante:

$$
A_{i}=A_{i} \cdot \frac{r_{i}}{r_{i}} \cdot \exp \left(-\alpha \cdot\left(r_{i}-r_{i}\right)\right)
$$

Cette expression permet l'estimation du signal d'accélération au point $M$ d'après le signal mesuré au point $M_{1}$. Le rapport $r / r$ rend compte de l'atténuation géométrique (expansion du front d'onde), alors que le facteur $\exp \left[-\alpha \cdot\left(r_{-}-r\right)\right]$ donne l'atténuation matérielle entre $M$ et $M$. Cette expression est toutefois trop simple pour décrire parfaitement les phénomènes de propagation [12]

\section{2}

\section{Propagation d'onde et comportement}

Les mesures d'accélération réalisées indiquent que, pour les accéléromètres situés à mi-profondeur, les ondes créées sont des ondes longitudinales (seules les composantes radiales d'accélération sont non nulles [12], voir figure 5). A partir de cette hypothèse et en approchant le comportement du matériau par un modèle viscoélastique linéaire, il est possible d'écrire les équations de la mécanique (équilibre et comportement) sous une forme assez simple. La résolution analytique du problème de propagation peut alors se faire dans le domaine fréquentiel. Les jeux de paramètres de comportement optimaux sont déterminés à partir de ces solutions analytiques.

\section{3}

\section{Solution simplifiée}

Une première hypothèse relativement forte est proposée : seuls les termes radiaux de contrainte et de déplacement son conservés ([12], [13]), et l'équation de propagation simplifiée s'écrit sous la forme suivante:

$$
\operatorname{div}(\sigma(r, t))=\frac{\partial \sigma_{r r}(r, t)}{\partial r}+\frac{2}{r} \cdot \sigma_{r r}(r, t)=\rho \cdot \frac{\partial^{2} u_{r}(r, t)}{\partial t^{2}}
$$

où $\sigma$ représente la contrainte, $\mathrm{r}$ la distance à la source et u le déplacement.

En supposant le comportement viscoléastique linéaire et en réalisant l'approximation $\mathrm{E}=\lambda+2 \mu$ (pour simplifier l'écriture), il est possible de relier aisérnent les paramètres de contrainte et de déformation à l'aide d'une relation du type :

$$
\sigma_{\pi r}^{*}(r, \omega)=E^{*}(\omega) \cdot \varepsilon_{\pi}^{*}(r, \omega)
$$

où $\sigma^{*}(r, \omega)$ et $\varepsilon^{*}(r, \omega)$ représentent respectivement le spectre complexe de la contrainte $\left(\sigma_{\pi}\right)$ et de la défor- mation $\left(\varepsilon_{-}\right)$à la distance $r$ et la pulsion $\omega$, et $E^{*}(\omega)$ est le module complexe qui dépend du modèle viscoélastique choisi.

La combinaison entre équation de propagation et équation de comportement donne, dans le domaine spectral, l'équation en déplacement suivante:

$$
\frac{\partial^{2} u_{r}^{\prime}(r, \omega)}{\partial r^{2}}+\frac{2}{r} \frac{\partial u_{r}^{*}(r, \omega)}{\partial r}=\frac{-\rho \omega^{2}}{E^{\prime}(\omega)} \cdot u_{r}^{*}(r, \omega)
$$

dont la solution s'écrit sous la forme:

$$
u^{\prime}(r, \omega)=\frac{U_{0}(\omega)}{r} \cdot \exp (i \cdot \xi(\omega) \cdot r)
$$

oủ $\xi$ est le nombre d'onde complexe, fonction de la pulsation $\omega$, tel que :

$$
\xi^{2}(\omega)=\frac{\rho \cdot \omega^{2}}{E^{*}(\omega)}
$$

L'expression de la solution du problème (équation (2)) fait clairement apparaître l'atténuation à travers deux facteurs :

- l'atténuation géométrique qui rend compte de l'expansion du front d'onde sphérique et qui est inversement proportionnelle à $\mathrm{r}$ (distance à la source) mais indépendante de la fréquence ;

- l'atténuation matérielle qui apparaît dans le terme d'amortissement visqueux (partie imaginaire de $\xi(\omega)$ ) et qui traduit la dissipation énergétique au sein-même du milieu.

C'est cette solution analytique qui est utilisée dans [12] et [13] pour réaliser des simulations analytiques de propagation d'ondes sphériques. Dans le présent article, cette solution est comparée à une solution plus complète qui prend en compte l'ensemble des composantes du tenseur de déformation (voir paragraphe suivant).

\section{4}

\section{Solution complète (ondes P)}

\section{4 .4}

\section{Tenseurs de déformation et de contrainte}

L'hypothèse précédente réduit le tenseur de déformation au seul $1^{\text {er }}$ terme diagonal $\varepsilon_{\pi}$. En supposant que le déplacement se fait uniquement suivant la direction radiale (ce qui est vérifié expérimentalement), le tenseur de déformation complet s'écrit de la manière súvante :

$$
\varepsilon=\left[\begin{array}{ccc}
\frac{\partial u_{r}}{\partial r} & \frac{1}{2 r} \frac{\partial u_{r}}{\partial \theta} & \frac{1}{2 r \cdot \sin \theta} \frac{\partial u_{r}}{\partial \theta} \\
\frac{1}{2 r} \frac{\partial u_{r}}{\partial \theta} & \frac{u_{r}}{r} & 0 \\
\frac{1}{2 r \cdot \sin \theta} \frac{\partial u_{r}}{\partial \theta} & 0 & \frac{u_{r}}{r}
\end{array}\right]
$$


Le tenseur de contrainte peut donc être déterminé aisément de la façon suivante :

$$
\begin{gathered}
\sigma_{r r}=(\lambda+2 \mu) \frac{\partial u_{r}}{\partial r}+\lambda \frac{2 u_{r}}{r} \\
\sigma_{\theta \theta}=\lambda\left(\frac{\partial u_{r}}{\partial_{r}}+\frac{2 u_{r}}{r}\right)+2 \mu \frac{u_{r}}{r} \\
\sigma_{\varphi \varphi}=\lambda\left(\frac{\partial u_{r}}{\partial_{r}}+\frac{2 u_{r}}{r}\right)+2 \mu \frac{u_{r}}{r} \\
\sigma_{r \theta}=\frac{\mu}{r} \frac{\partial u_{r}}{\partial \theta} ; \sigma_{r \varphi}=\frac{\mu}{r \cdot \sin \theta} \frac{\partial u_{r}}{\partial \varphi} \text { et } \sigma_{\theta \varphi}=0
\end{gathered}
$$

Ces expressions sont notablement plus complètes que celles du paragraphe précédent et tiennent compte de déformations de distorsion ou de type membranaire.

\section{Fanin}

\section{Solution du problème complet}

Aucune dépendance en $\theta$ et $\varphi$ n'est prise en compte (et $\mathrm{E}=\lambda+2 \mu$ ), les équations d'êquilibre en coordonnées sphériques se réduisent donc à l'équation différentielles suivante :

$$
\frac{\partial^{2} u_{r}}{\partial r^{2}}+\frac{2}{r} \frac{\partial u_{r}}{\partial r}-\frac{2}{r^{2}} u_{r}=\frac{\rho}{E} \frac{\partial^{2} u_{r}}{\partial t^{2}}
$$

La solution de l'équation différentielle (3) est donc la suivante :

$$
U^{*}(r, \omega)=\frac{U_{0}^{*}(\omega)}{r} \cdot\left(\xi(\omega)+\frac{i}{r}\right) \exp (i \cdot \xi(\omega) \cdot r)
$$

La solution complète (4) est légèrement différente de la solution simplifiée (2) et comporte un terme supplémentaire en $1 / r^{2}$ qui décroît rapidement quand $r$ augmente. Les différences entre ces deux solutions sont évaluées dans le paragraphe suivant.

\section{6}

\section{Simulation d'après les signaux expérimentaux}

\section{1}

\section{Choix des modèles rhéologiques}

Les modèles viscoélastiques linéaires choisis permettent de traduire différents types de dépendance atténuation-fréquence [12, 13]. Ils sont représentés à la figure 14 ainsi que les courbes d'atténuation qui les caractérisent. Les modules complexes associés à ces modèles permettent de déterminer l'expression de leurs atténuations :

$$
\begin{aligned}
& Q_{\text {Max }}^{-1}=\frac{E}{\omega \cdot \eta} \text { et } Q_{\text {Kel }}^{-1}=\frac{\omega \cdot \eta}{E} \\
& Q_{Z e n}^{-1}=\frac{\omega \cdot \eta \cdot E}{E_{v} \cdot\left(E+E_{v}\right)+\omega^{2} \eta^{2}}
\end{aligned}
$$

respectivement pour les modèles de Maxwell, KelvinVoigt et Zener ( $\mathrm{E}$ : module d'Young, $\eta$ : coefficient de viscosité, $E_{v}$ : module « visqueux $»$ et $\omega$ : pulsation).

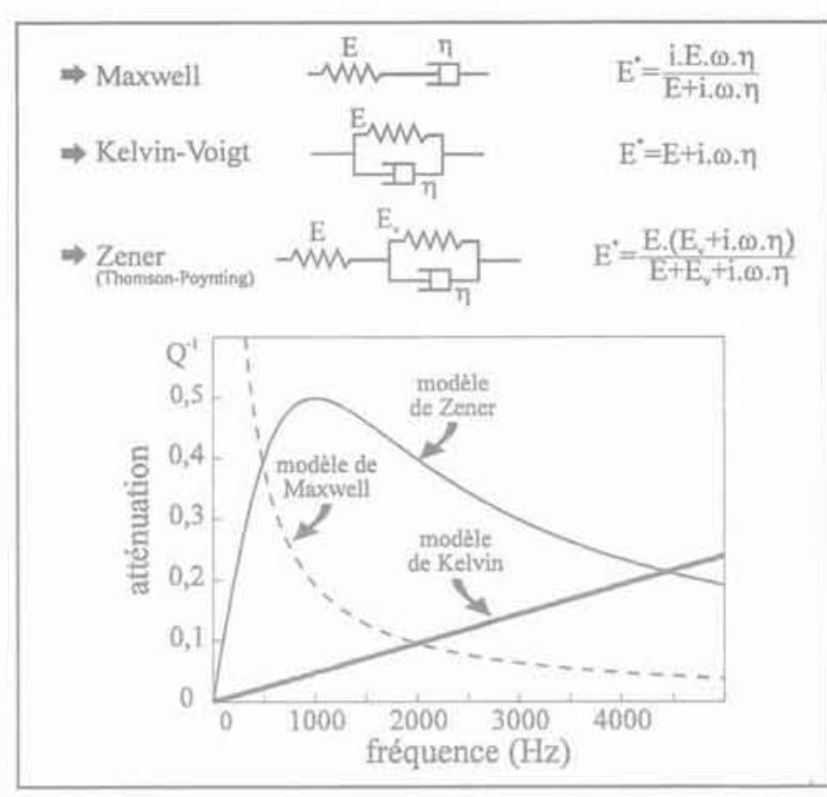

FiG.14 Différents modèles rhéologiques et courbes d'atténuation associées. Fineological models and related attenuation curves.

\section{2}

Simulations réalisées

\section{4}

\section{Paramètres de comportement}

Pour les modèles de Maxwell et de Kelvin-Voigt, les deux paramètres de comportement sont le module d'Young E et le coefficient de viscosité $\eta$. E peut être aisément calculé pour des ondes de compression d'après la célérité de ces ondes, soit $C$, en utilisant la relation :

$$
C=\sqrt{\frac{\lambda+2 \mu}{\rho}}=\sqrt{\frac{E \cdot(1-v)}{\rho \cdot(1+v) \cdot(1-2 v)}}
$$

où $\rho$ est la masse volumique du sable $\left(\rho=1650 \mathrm{~kg} / \mathrm{m}^{3}\right)$ et $v$ le coefficient de Poisson.

La célérité des ondes est déterminée expérimentalement soit $\mathrm{C}=435 \mathrm{~m} \cdot \mathrm{s}^{-1}$. En considérant l'approximation $E=\lambda+2 \mu$ (pour simplifier l'écriture), le module d'Young du sable vaut donc $E=310 \mathrm{MPa}$.

\section{6ar?}

\section{Simulations d'après la solution simplifiée}

A partir d'un signal mesuré à la distance $r$ de la source, la solution simplifiée (équation (2)) donne l'accélération à la distance $\mathrm{r}$, à l'aide de la relation suivante:

$$
\frac{a_{m}^{*}\left(r_{j}, \omega\right)}{a_{m}^{*}\left(r_{i}, \omega\right)}=\frac{r_{i}}{r_{j}} \cdot \exp \left[i \cdot \xi(\omega) \cdot\left(r_{i}-r_{i}\right)\right]
$$

où $a_{m}^{*}(r, \omega)$ représente le spectre complexe de l'accélération mesurée à la distance $r_{1}$ (idem pour $r$ ). 
Par transformation de Fourier inverse, l'accélération simulée à la distance $r$ peut être calculée d'après cette expression :

$$
a_{m}^{\operatorname{sim}}\left(r_{j}, t\right)=\int_{-\infty}^{+\infty} a_{m}^{*}\left(r_{i}, \omega\right) \cdot \frac{r_{1}}{r_{j}} \cdot \exp \left[i \cdot \xi(\omega)\left(r_{i}-r_{i}\right)\right] \exp (i \cdot \omega, t) \cdot d \omega
$$

La comparaison des simulations obtenues d'après cette résolution analytique avec les résultats expérimentaux est faite d'après les signaux temporels d'accélération. Le choix du modèle viscoélastique le mieux adapté dépend des caractéristiques de ce modèle. L'expression de l'atténuation $\left(\mathrm{Q}^{-1}\right)$ est très différente suivant le type de modèle choisi (voir [12] et figure 14):

- $Q^{-1}$ est proportionnelle à la fréquence pour le modèle de Kelvin-Voigt :

- $Q^{-1}$ est inversement proportionnelle à la fréquence pour le modèle de Maxwell :

- l'atténuation peut présenter des variations plus complexes : effet coupe-bande pour le modèle de Zener (ressort en série avec cellule de Kelvin)

\subsection{8}

\section{Simulations d'après la solution complète}

La solution complète définie par l'équation (4) permet de la même manière que précédemment d'écrire une relation entre signal mesuré à la distance $r_{\text {, }}$ et signal simulé à la distance $r_{j}$. Cette relation s'exprime de la manière suivante:

$$
\frac{a_{m}^{*}\left(r_{j}, \omega\right)}{a_{m}^{*}\left(r_{i}, \omega\right)}=\frac{r_{i}^{2}}{r_{j}^{2}} \cdot \frac{r_{j} \cdot \xi(\omega)+i}{r_{i} \cdot \xi(\omega)+i} \cdot \exp \left[i \cdot \xi(\omega) \cdot\left(r_{j}-r_{i}\right)\right]
$$

Cette expression comporte un terme proportionnel au rapport des carrés des distances à la source qui distingue cette solution de la précédente. Or ce terme doit, d'après sa forme, décroître rapidement avec la distance. C'est ce qu'indiquent les courbes de la figure 15: près de la source, solution simplifiée et solution complète conduisent à des simulations très différentes. Lorsque la distance à la source augmente, la différence entre les deux solutions devient faible.
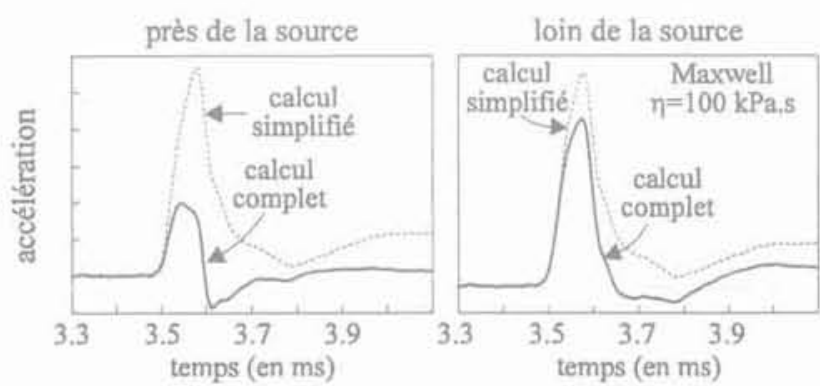

FIG. 15 Simulations viscoélastiques: comparaison solutions simplifiée et complète.

Viscoelastic modelling : comparison between simplified and complete solutions.

D'après les valeurs de paramètres mécaniques obtenues dans [12], la différence entre solutions simplifiée et complète (équations (2) et (4)) devient faible lorsque la distance à la source dépasse $0,05 \mathrm{~m}$. Comme c'est le cas pour tous les accéléromètres utilisées, c'est la solution simplifiée qui sera retenue.

\section{Comparaisons avec les résultats expérimentaux}

Les résultats proposés à la figure 16 permettent de comparer les mesures expérimentales avec des simulations issues des modèles de Maxwell et Kelvin. L'accélération de référence est le signal d'accélération délivré par le capteur 4.

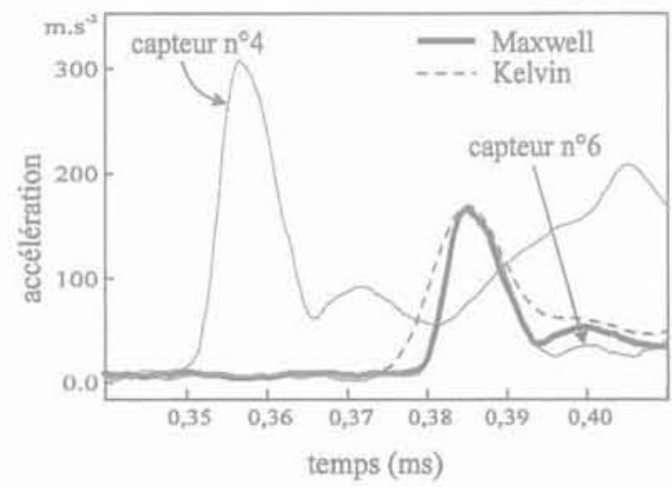

FiG. 16 Simulations viscoélastiques d'après signaux expérimentaux.

Viscoelastic modelling from experimental signals.

La coïncidence entre simulation et accélération mesurée (capteur 6) est peu satisfaisante pour le modèle de Kelvin mais est en revanche très bonne pour le modèle de Maxwell. Les coefficients de viscosité $\eta$ utilisés dans les exemples de la figure 16 sont les suivants : $\eta_{\text {Ket }}=1000$ Pa.s et $\eta_{\text {Max }}=150000$ Pa.s.

\section{3}

\section{Paramètres de comportement optimaux}

Pour déterminer les paramètres de comportement optimaux $\left(\mathrm{E}_{\text {, et }} \eta\right.$ ) pour le modèle de Zener (voir Fig. 14), il est nécessaire d'apprécier quantitativement la qualité de la simulation réalisée. Il est par exemple possible de déterminer l'erreur quadratique entre signaux mesurés et signaux simulés. En considérant l'intervalle de temps $\left[t_{1} ; t_{2}\right]$ autour du premier pic d'accélération, la fonctionnelle $J(E, \eta)$, définie comme l'erreur quadratique entre l'accélération mesurée $a_{m}(r)$ et l'accélération calculée $a_{m}{ }^{\operatorname{sim}}(r)$ peut s'écrire :

$$
J\left(E_{v}, \eta\right)=\frac{1}{J_{0}} \cdot \int_{t_{1}}^{t_{2}}\left(a_{m}\left(r_{j}, t\right)-a_{m}^{\operatorname{sim}}\left(r_{j}, t\right)\right)^{2} \cdot d t
$$

La minimisation de $J\left(E_{v}, \eta\right)$ donne les paramètres de comportement optimaux $\left(E_{\text {yop }}, \eta_{\text {opp }}\right)$. La courbe de la figure 17 donne les valeurs de la fonctionnelle $J\left(E_{v}, \eta\right)$ pour un module $E_{v} \in[0 ; 50 \mathrm{MPa}]$ et une viscosité $\eta \in[130 ; 160 \mathrm{kPa} . \mathrm{s}]$ (pour les capteurs 4 et 6 ).

L'erreur minimale $J_{\min }$ est obtenue pour un module $E_{v}=25,5 \mathrm{MPa}$ et une viscosité $\eta=146 \mathrm{kPa}$.s. Le module " visqueux $»$ optimal $E_{v \text {, apt }}$ est très faible, et la viscosité 
optimale $\eta_{\text {opt }}$ est très proche de la valeur choisie pour le modèle de Maxwell. Avec ces deux paramètres de comportement, les simulations obtenues avec les modèles de Zener et Maxwell sont très proches. Les deux modèles doivent ètre comparés d'un point de vue quantitatif.

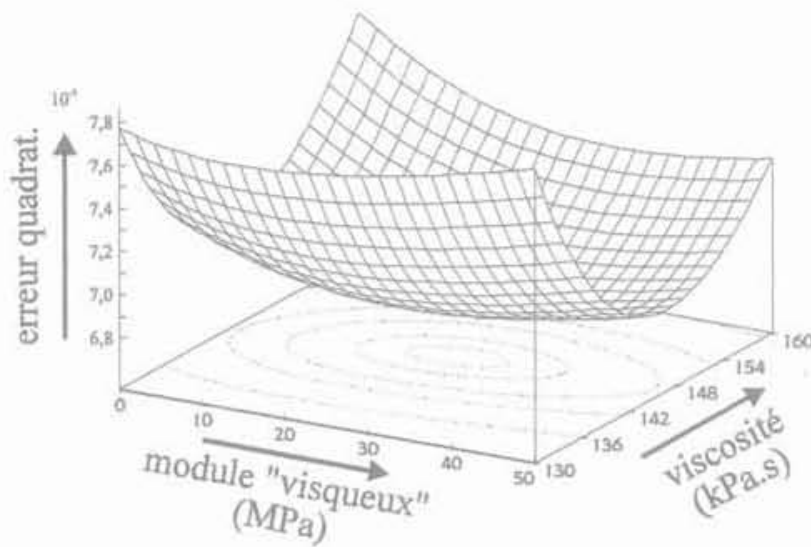

FiG. 17 Détermination des paramètres de comportement optimaux (modèle de Zener).

Determination of optimal behaviour parameters (Zener model).

En calculant l'erreur J( $\eta$ ) pour le modèle de Maxwell, la valeur optimale correspond à une viscosité optimale $\eta_{\text {opt }}=144 \mathrm{kPa}$.s. Cette valeur diffère assez peu de la valeur déterminée empiriquement précédemment $(\eta=150 \mathrm{kPa}$.s, voir figure 16). La valeur minimale de $J(\eta)$ pour le modèle de Maxwell est JMax $=7,02 \cdot 10^{-3}$. Pour le modèle de Zener, elle est un peu plus faible et vaut JZen $=6,80 \cdot 10^{-3}$. Le modèle de Zener donne donc une simulation légèrement plus proche des résultats expérimentaux que le modèle de Maxwell.

\section{4}

\section{Vitesse de groupe, vitesse de phase}

Si $\xi(\omega)$ est le nombre d'onde complexe, les expressions classiques des vitesses de phase et de groupe deviennent complexes. Les parties réelles de ces expressions correspondent aux termes de déphasage et caractérisent la vitesse de phase $V_{\phi}$ et la vitesse de groupe $V_{g}$. Ces expressions prennent alors la forme suivante:

$$
V_{\Phi}=\operatorname{Re}\left[\frac{\omega}{\xi(\omega)}\right] \text { et } V_{g}=\operatorname{Re}\left[\frac{\mathrm{d} \omega}{d \xi(\omega)}\right]
$$

où Re représente la partie réelle d'une variable complexe.

La détermination des paramètres de comportement viscoélastique permet de calculer les vitesses de phase et de groupe théoriques pour les capteurs situés à miprofondeur. Les courbes de la figure 18 donnent les valeurs de $V_{\phi}$ et $V_{q}$ pour une simulation viscoélastique de Maxwell. Sur la figure 18 , ces courbes sont comparées avec les valeurs estimées expérimentalement d'après les analyses temps-fréquence réalisées précé- demment. Ceci permet une comparaison dans l'intervalle de fréquence $[0 \mathrm{~Hz} ; 5500 \mathrm{~Hz}]$. Au-delà de cet intervalle, les valeurs théoriques et expérimentales coincident parfaitement.

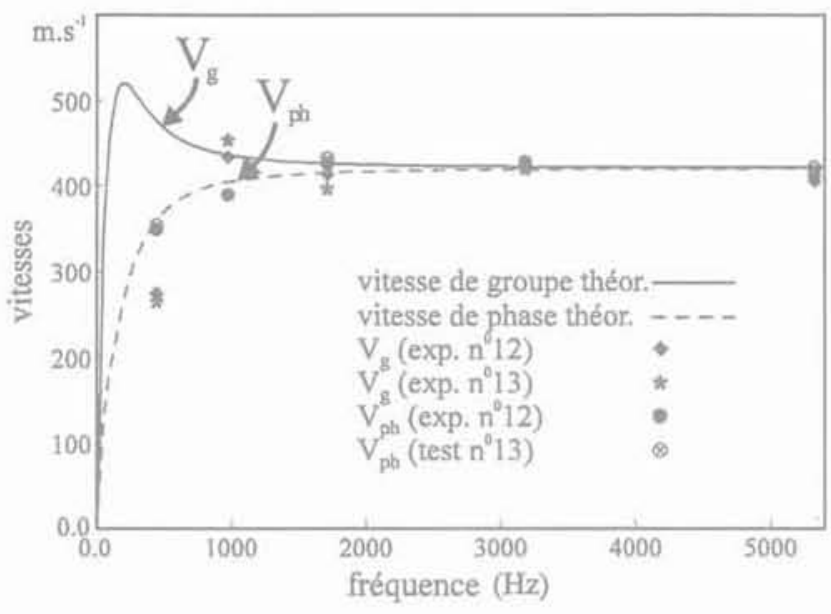

fIG 18 Comparaisons des vitesses de groupe et de phase (expérimentales et théoriques). Group and phase velocities comparison (experimental vs theoretical).

Entre 0 et $5500 \mathrm{~Hz}$, la comparaison des valeurs théoriques et expérimentales mène aux conclusions suivantes:

- au-dessus de $1500 \mathrm{~Hz}$ : les vitesses de phase et de groupe sont quasiment identiques ; il y a une bonne coîncidence entre valeurs théoriques et expérimentales;

- à $975 \mathrm{~Hz}$ : la vitesse de groupe est supérieure à la vitesse de phase aussi bien pour les valeurs théoriques que pour les valeurs expérimentales; la coincidence entre les deux types de résultats est assez bonne ;

- à $440 \mathrm{~Hz}$ : la vitesse de groupe théorique est plus grande que la vitesse de phase théorique; les vitesses de phase expérimentales ne sont pas très différentes des vitesses de phase théoriques mais pour les vitesses de groupe la différence est très grande. En fait, les longueurs d'ondes aux faibles fréquences ( $<<1000 \mathrm{~Hz}$ ) sont importantes et les vitesses de groupe sont fortement sous-estimées.

Comme indiqué précédemment, un effet de guide d'ondes important apparait aux faibles fréquences. La sous-estimation de la vitesse de phase n'est pas très importante, mais pour les vitesses de groupe l'importance des réflexions d'ondes est grande (effet de guide d'ondes). Pour les faibles fréquences, la dispersion géométrique doit être prise en compte. C'est une des principales difficultés des expérimentations dynamiques en milieu fini.

\section{Simulations à différentes distances de la source}

Les précédentes simulations sont toutes réalisées pour la même paire de capteurs ( $n^{\circ} 4$ et $n^{\circ} 6$ ). Il est nécessaire de réaliser d'autres simulations pour différentes paires de capteurs pour étudier l'influence de la 
distance à la source et du niveau d'accélération. La détermination précise des paramètres de comportement permet la comparaison des différents résultats.

Pour le modèle Maxwell, quatre paires de signaux " excitation-réponse » sont tirées des mesures d'accélération réalisées par les capteurs situés à mi-profondeur $(2 / 4 ; 4 / 6 ; 6 / 8$ et $8 / 10)$. L'erreur quadratique $J(\eta)$ est déterminée dans chaque cas pour estimer la valeur de viscosité optimale $\eta$. Comme indiqué dans le tableau IV, les résultats sont très différents d'un cas à l'autre

Pour le modèle de Maxwell, l'atténuation $Q^{-1}$ est reliée et aux paramètres de comportement par la relation:

$$
Q^{-1}=\frac{E}{\eta \cdot \omega}
$$

En utilisant un modèle viscoélastique linéaire, les solutions analytiques proposées ne tiennent compte ni de la dépendance amplitude-atténuation [12] ni des possibles variations de l'amortissement avec la profondeur [17]. Ces deux phénomènes peuvent expliquer que l'atténuation matérielle soit forte pour la première paire de capteurs et quasiment nulle pour les deux derniers capteurs (8 et 10). En effet, dans ce dernier cas, l'atténuation géométrique réduit à elle seule l'amplitude d'accélération d'environ $20 \%$, l'atténuation matérielle serait alors quasiment inexistante.

Une quantification partielle de l'atténuation est réalisée (tableau IV). La modélisation proposée n'est toutefois pas assez complète pour rendre compte des variations de l'atténuation (en fonction de la distance, de l'amplitude...). Des simulations numériques plus complexes sont proposées dans [17] afin d'obtenir une description globale cohérente des phénomènes de propagation et d'atténuation.
TABleauiv Valeurs optimales de viscosité (Maxwell) pour les simulations à différentes distances de la source.

Optimal (Maxwell) viscosity values for simulations at different distances from the source.

\begin{tabular}{c|c|c|c}
\hline \multicolumn{2}{c|}{ Capteur } & \multicolumn{2}{c}{ Paramètres de comportement } \\
\hline entrée & sortie & viscosité & $J(\eta)$ \\
\hline 2 & 4 & $39 \mathrm{kPa} . \mathrm{s}$ & $1,63 \cdot 10^{-7}$ \\
\hline 4 & 6 & $144 \mathrm{kPa} \cdot \mathrm{s}$ & $7,02.10^{-1}$ \\
\hline 6 & 8 & $154 \mathrm{kPa} . \mathrm{s}$ & $2,22.10^{-2}$ \\
\hline 8 & 10 & $+\infty$ pas d'atténuation matérielle \\
\hline
\end{tabular}

\section{8}

\section{Conclusion}

Un dispositif expérimental original est proposé pour étudier la propagation d'ondes en milieu centrifugé. Une analyse approfondie des divers phénomènes régissant la propagation est réalisée : réflexions, dispersion, atténuation. La modélisation du champ d'onde sphérique en viscoélasticité linéaire permet une description analytique complète de la propagation pour les essais de chute de masse. Les simulations réalisées permettent d'apprécier globalement les phénomènes d'atténuation et de dispersion. Toutefois, la mise en cuvre de modèles plus complets semble nécessaire afin d'aboutir à une description plus réaliste des phénomènes d'atténuation.

\section{Bibliographie}

[1] Abe S., Kobayashi Y.. Ikawa T. - Sejsmic characteristics of the weight-dropping source. Jal of Physics Earth, vol. 38, 1990, p. $189-212$

[2] Arulanandan K., Canclini J., Anandarajah A. - Simulation of earthquake motions in the centrifuge. Jal of Geotechnical Engineering, ASCE, vol. 108, n² GI5, mai 1982, p. 730-742.

[3] Cheney J.A., Brown R.K., Dhat N.A., Hor O.Y.Z. - Modeling free-field conditions in centrifuge models. Jal of Geotechnical Engineering, vol. 116, $\mathrm{n}^{\circ} \mathrm{9}$, 1990, p. 1347-1367.

[4] Coe C.J., Prevost J.H., Scanlan R.H. Dynamic stress wave reflections/attenuation : eartquake Engineering and Structural Dynamics, vol. 13, 1985, p. 109-128.

[5] Farnbach J.S. - The complex enveloppe in seismic signal analysis. Bulletin of the Seismological Society of America vol. 65 , $\mathrm{n}^{\circ} 4$. 1975, p. 951-962.

[6] Flandrin P., Sessarego J.P. - Méthodes temps-fréquence en acoustique. Colloque de Physique C2, Tome 51, 1990, p. 707-716.

[7] Heitz J.-F., Bonnet G. - Effets non linéaires en dynamique des sols : essais in situ. Revue Francaise de Géotechnique, $n^{\circ} 46$, janvier 1989, p. 19-30.

[8] Jaffard S. - Alcorithmes de transformation en ondelettes. Annales des Ponts et Chaussées, 1989, p. 10-30.

[9] Lightill M.J. - Group velocity. Jal of the Institute of Maths and its Applications. vol. 1, 1965, p. 1-28

[10] Luong M.P. - Experimental methods in eartquake engineering. In Recent advances in earthquake engineering \& structural dynamics, éd. V. Davidovici, Presses de I'ENPC. Paris, 1992.

[11] Luong M.P. - Centrifuge simulation of Rayleigh waves in soils using a dropball arrangement : Dynamic Geotechnical Testing II, ASTM STP 1213, éd. R.J. Ebelhar, V.P. Drnevich \& B.L. Kutter. Philadelphie, 1995.

[12] Semblat J.F. - Sols sous sollicitations dynamiques et transitoires : réponse dynamique aux barres de Hopkinson. propagation d'ondes en milieu centrifugé. Thèse de Doctorat de l'École polytechnique, Rapport Études et Recherches des LPC, $\square^{\circ}$ GT60. Laboratoire Central des Ponts et Chaussées. Paris, mars 1995, $206 \mathrm{p}$.
[13] Semblat J.-F., Luong M.P., Thomas J.-J - Drop-ball arrangement for centrifuge experiments. 5th SECED Conference on European Seismic Design Practice, Chester, UK, 26-27 octobre 1995, p. 567-575

[14] Semblat J.-F., Luong M.P. Réponse dynamique des sols : essais aux barres de Hopkinson: Revue Française de Géotechnique, 79, 1997, p. 19-30.

[15] Semblat J.F. - Amortissement et dispersion des ondes : points de vue physique et numérique. Revue Française de Génie Civil, vol. 2, n 1, 1998, p.91-111.

[16] Semblat J.F. - Rheological interpretation of Rayleigh damping. Journal of sound and Vibration, vol. 206, n 5, 1997, p. $741-744$.

[17] Semblat J.F Brioist J J Luong M.P. Numerical modelling of wave propagation in centrifuged medium. Int. Conf Centrifuge 98. Tokyo, 23-25 septembre 1998.

[18] Tribolet J.M. - A new phase unwrapping algorithm, IEEE Transcactions on Acoustics, Speech and Signal Processing. vol. $25, n^{\circ} 2,1977$, p. $170-177$

[19] Ulrych T.J. - Application of homomor phic deconvolution to seismology, Geophysics, vol. $36, \pi^{\circ} 4.1971$, p. $650-660$. 